\title{
Diacronie
}

Studi di Storia Contemporanea

$N^{\circ} 33,1 \mid 2018$

Guerra e pace

\section{Nicola De Ianni, Il calcio italiano 1898-1981. Economia e potere}

\section{Matteo Anastasi}

\section{OpenEdition}

Journals

\section{Edizione digitale}

URL: http://journals.openedition.org/diacronie/7486

DOI: 10.4000/diacronie.7486

ISSN: 2038-0925

\section{Editore}

Association culturelle Diacronie

Notizia bibliografica digitale

Matteo Anastasi, « Nicola De lanni, I/ calcio italiano 1898-1987. Economia e potere », Diacronie [Online], N 33, 1 | 2018, documento 9, Messo online il 29 mars 2018, consultato il 23 septembre 2020. URL : http://journals.openedition.org/diacronie/7486 ; DOI : https://doi.org/10.4000/diacronie.7486 


\title{
Diacronie
}

Studi di Storia Contemporanea

\section{$33,1 / 2018$}

Guerra e pace: declinazioni politiche, sociali e culturali del conflitto in età contemporanea

\section{RECENSIONE: Nicola DE IANNI, Il calcio italiano 1898-1981. Economia e potere, Soveria Mannelli, Rubbettino, 2015, 245 pp.}

\author{
A cura di Matteo ANASTASI
}

Per citare questo articolo:

ANASTASI, Matteo, «RECENSIONE: Nicola DE IANNI, Il calcio italiano 1898-1981. Economia e potere, Soveria Mannelli, Rubbettino, 2015, 245 pp.», Diacronie. Studi di Storia Contemporanea : Guerra e pace: declinazioni politiche, sociali e culturali del conflitto in età contemporanea, 33, 1/2018, 29/03/2018,

URL: < http://www.studistorici.com/2018/03/29/anastasi_numero_33/>

Diacronie Studi di Storia Contemporanea $\rightarrow$ http://www.diacronie.it Rivista storica online. Uscita trimestrale.

redazione.diacronie@hotmail.it

Comitato di direzione: Naor Ben-Yehoyada - João Fábio Bertonha - Christopher Denis-Delacour - Maximiliano Fuentes Codera Anders Granås Kjøstvedt - John Paul Newman - Deborah Paci - Niccolò Pianciola - Spyridon Ploumidis - Wilko Graf Von Hardenberg

Comitato di redazione: Jacopo Bassi - Luca Bufarale - Gianluca Canè - Luca G. Manenti - Fausto Pietrancosta - Alessandro Salvador - Matteo Tomasoni - Luca Zuccolo modifiche dei contenuti, di riconoscere la paternità dell'opera e di condividerla allo stesso modo. La citazione di estratti è comunque sempre autorizzata, nei limiti previsti dalla legge. 


\title{
9/ RECENSIONE: Nicola DE IANNI, Il calcio italiano 1898-1981. Economia e potere, Soveria Mannelli, Rubbettino, 2015, 245 pp.
}

\author{
A cura di Matteo ANASTASI
}

Il volume di Nicola De Ianni, professore associato di Storia economica presso il Dipartimento di Scienze economiche e statistiche dell'Università Federico II di Napoli, intende studiare «il valore e il peso del denaro prima dell'assoluta trasformazione del calcio in settore economico» ${ }^{1}$. Per adempiere tale intento, prende in esame un arco temporale molto esteso, che conosce i suoi estremi cronologici nel 1898 - anno della nascita della Federazione Italiana del Football (FIF), la futura FIGC - e nel 1981 - anno successivo al primo scandalo calcioscommesse e momento conclusivo della presidenza di Artemio Franchi. La pubblicazione del saggio è stata preceduta da una serie di (propedeutici alla stesura del lavoro) seminari organizzati nell'ambito del corso di Storia dell'industria all'Università Federico II che hanno visto come relatori figure a vario titolo protagoniste del panorama calcistico italiano: dal procuratore Dario Canovi al dirigente Marco Fassone, passando per Corrado Ferlaino, già presidente della Società Sportiva Calcio Napoli, e Marcello Lippi, allenatore della Nazionale campione del mondo nel 2006.

Per De Ianni è un esordio nel campo della storia dello sport. La sua ampia produzione scientifica si è concentrata, negli anni precedenti, soprattutto sul ruolo di specifici attori economici della scena pubblica italiana - si pensi, in particolare, ai volumi Il ministro soldato. Vita di Guido Jung e Banca e mercato nell'opera di Antonio Confalonieri - e su studi di storia economica locale, come nel caso di Operai e industriali a Napoli tra Grande Guerra e crisi mondiale (1915-1929), Per la storia dell'industria a Napoli e Banco di Napoli spa (1991-2002). Un decennio difficile².

Il testo in esame si compone di tre parti, precedute da una premessa nella quale è fornito un quadro di riferimento sull'evoluzione organizzativa del governo del calcio e sulla sua

\footnotetext{
${ }^{1}$ DE IANNI, Nicola, Il calcio italiano 1898-1981. Economia e potere, Soveria Mannelli, Rubbettino, 2015, p. 7.

2 ID., Operai e industriali a Napoli tra Grande Guerra e crisi mondiale (1915-1929), Genève, Librairie Droz, 1984; ID., Per la storia dell'industria a Napoli, Napoli, Istituto italiano per la storia delle imprese, 1990; ID., Banca e mercato nell'opera di Antonio Confalonieri, Roma, Carocci, 2001; ID., Banco di Napoli spa (1991-2002). Un decennio difficile, Soveria Mannelli, Rubbettino, 2007; ID., Il ministro soldato. Vita di Guido Jung, Soveria Mannelli, Rubbettino, 2009.
} 
periodizzazione. Successivamente, nella prima parte, si passa ad analizzare gli aspetti macroeconomici attraverso la ricostruzione di una serie storica con ricavi, spese e deficit. Lo studio è effettuato separando il primo cinquantennio d'attività del calcio italiano (1898-1948) - la cui tendenza dei ricavi può essere, precisa De Ianni, «con buona approssimazione, soltanto stimata» ${ }^{3}$ - dal trentennio 1951-1981, in cui il giro d'affari intorno al pallone cresce esponenzialmente con l'introduzione del Totocalcio, la vera innovazione economica del dopoguerra. L'iniziale ripartizione dei ricavi stabilita nel 1946 - 45\% delle entrate destinato al montepremi, $20 \%$ alla SISAL, $7 \%$ al CONI, $11 \%$ allo Stato e $5 \%$ al Ministero dell'Interno per essere distribuito in beneficienza - è rapidamente rivista in virtù delle crescenti pretese di Stato e CONI. Quest'ultimo, nel 1948, assume in proprio la gestione del gioco, estromettendo la SISAL. In aprile, con un decreto poi trasformato in legge, lo Stato destina a sé il $23 \%$ dei ricavi, aumentando al $48 \%$ la quota riservata ai vincitori, riducendo al $4 \%$ quella delle ricevitorie e garantendo il $25 \%$ al CONI. È l'inizio di un processo che porta lo Stato, nel 1954, a superare la quota del CONI. La prima parte si chiude con un interessante affresco su nascita ed evoluzione di dinamiche economiche divenute sentire e leggere quotidiano degli appassionati di calcio e non solo: premi, stipendi, ingaggi, contratti pluriennali, diritti alle prestazioni, plusvalenze e minusvalenze

Nella seconda parte dello studio sono presi in considerazione gli aspetti microeconomici mediante l'individuazione di tre figure chiave: presidenti, allenatori e dirigenti. Dopo un'analisi del passaggio dai clubs alle società per azioni (1898-1966) e della nascita delle società senza fine di lucro (1966-1981), necessaria a evitare "alla gestione calcistica le rigidità del codice civile» ${ }^{4}$, si tracciano i profili economici dei più incisivi presidenti del calcio italiano. Dal confronto piemontese degli anni Venti fra Enrico Marone Cinzano (1895-1968), patron del Torino, ed Eduardo Agnelli (1892-1935), presidente alla Juventus, si passa alle figure di Umberto Lenzini (1912-1987) e Corrado Ferlaino (1931), presidenti dei primi scudetti di, rispettivamente, Lazio e Napoli, per arrivare ai protagonisti del calcio di provincia come Costantino Rozzi (1929-1994) ad Ascoli e Romeo Anconetani (1922-1999) a Pisa e chiudere con l'avvento al Milan di Silvio Berlusconi (1936), maggiore interprete della rivoluzione economica avvenuta nel calcio degli anni Ottanta. La seconda figura chiave individuata da De Ianni è quella dell'allenatore che "può usare le sue relazioni con i calciatori per favorire un ingaggio o addirittura trattare direttamente con procuratori agenti o dirigenti», precisando chiaramente che «tali trasformazioni dell'allenatore, che corrispondono a un aggiornamento della figura nel calcio industria, si sono svolte nel tempo e a fasi» $»^{5}$. Dai pionieri - William Garbutt (1883-1964), per oltre vent'anni in Italia sulle panchine di Genova, Roma e Napoli; Vittorio Pozzo (1886-1968), Commissario Tecnico della Nazionale

\footnotetext{
3 ID., Il calcio italiano 1898-1981, cit., p. 15.

4 Ibidem, p. 49.

5 Ibidem, p. 78.
} 
campione del mondo nel 1934 e nel 1938, e Arpad Weisz (1896-1944), campione d'Italia con l'Ambrosiana di Milano e due volte con il Bologna, poi deportato e tragicamente scomparso ad Auschwitz - a Giovanni Trapattoni (1939), «l'allenatore più rappresentativo e vincente della fase di transizione al calcio industria», capace, con la sua spiccata personalità, di «corrispondere perfettamente alle esigenze di un mondo che stava cambiando velocemente verso forme articolate di spettacolarizzazione» ${ }^{6}$. In ultimo la figura dei dirigenti e, in particolare, dei procuratori. Su tutti, Antonio Caliendo (1944) che, con la procura ottenuta da Giancarlo Antognoni nel 1972 per lo sfruttamento dell'immagine, vanta «l'indubbia primogenitura temporale» ${ }^{7}$ sulla professione di procuratore.

Nella terza e ultima parte del suo studio De Ianni si concentra sul potere economico dei presidenti federali che si sono susseguiti nell'ampio arco temporale preso in considerazione. Dai pionieri alla presidenza di Leandro Arpinati (1926-1933) - «cui toccò il compito di mettere la camicia nera al calcio» ${ }^{8}$ - fino ai presidenti al timone della FIGC dal secondo dopoguerra alla fine degli anni Settanta: Ottorino Barassi (1946-1958), Bruno Zauli (1958-59), Umberto Agnelli (19591961), Giuseppe Pasquale (1961-1967), Artemio Franchi (1967-1976 e 1978-1980) e Franco Carraro (1976-1978).

Pur disponendo già di diverse analisi sugli aspetti economici del football italiano e sul loro impatto sulla storia contemporanea del Paese - si pensi allo studio di Francesco Maria Varrasi ${ }^{9}$ - o settoriali, sul rapporto fra calcio e politica - il riferimento è, in particolare, a Simon Martin ${ }^{10}$ l'originalità del lavoro di De Ianni sta certamente nel tentativo di considerare il lungo periodo, circa novant'anni di storia, che ha segnato la trasformazione del calcio da mera attività sportiva a vera e propria "industria". A onor del vero un arco temporale ancora più ampio di quello analizzato da De Ianni è stato coperto dalla importante opera sul calcio di Guido Panico e Antonio $\mathrm{Papa}^{11}$, ma essa si è concentrata soprattutto sull'aspetto sociale e sulla capacità del pallone di plasmare usi e costumi di un Paese più che sulla sua rilevanza economico-finanziaria.

Per tali ragioni, e pur non avendo né le ambizioni né l'effettiva fattezza di un'opera di completezza assoluta sull'argomento trattato, lo studio di De Ianni apre una prima traiettoria -

\footnotetext{
${ }^{6}$ Ibidem, p. 92.

7 Ibidem, p. 94.

8 GHIRELLI, Antonio, Storia del calcio in Italia, Torino, Einaudi, 1954, p. 73.

9 Cfr. VARRASI, Francesco Maria, Economia, politica e sport in Italia (1925-1935). Spesa pubblica, organizzazioni specializzate, impianti ed espansione delle pratiche agonistiche amatoriali e "professionistiche" in un Paese a regime autoritario, Firenze, Bertelli, 1999.

10 Cfr. MARTIN, Simon, Calcio e fascismo. Lo sport nazionale sotto Mussolini, Milano, Mondadori, 2006 [Ed. originale: Football and Fascism. The national game under Mussolini, Oxford, Berg, 2004].

${ }^{11}$ Lo studio di Panico e Papa si compone di due volumi, l'uno il proseguio dell'altro, pubblicati a distanza di un decennio. Cfr. PANICO, Guido, PAPA, Antonio, Storia sociale del calcio in Italia. Dai club dei pionieri alla nazione sportiva (1887-1945), Bologna, Il Mulino, 1993; IID., Storia sociale del calcio in Italia. Dai campionati del dopoguerra alla Champions league (1945-2000), Bologna, Il Mulino, 2000.
} 
come scrive l'autore stesso nella prefazione - per «futuri approfondimenti da parte di altri studiosi» ${ }^{12}$.

${ }^{12}$ DE IANNI, Nicola, Il calcio italiano 1898-1981, cit., p. 7. 


\section{L'AUTORE}

Matteo ANASTASI (1989) è dottorando di ricerca in Scienze dell'Economia Civile presso la Libera Università Maria Santissima Assunta (LUMSA) di Roma con un progetto su "Fascismo, sport e identità nazionale. Gli stadi di calcio come veicolo di propaganda e strumento di consenso popolare". È cultore della materia in Storia Contemporanea presso l'Università Europea di Roma. I suoi interessi di ricerca sono relativi alla storia contemporanea e alla storia delle relazioni internazionali, con particolare riferimento alla storia della politica estera italiana e alla storia dello sport. Ł̀ socio della SISSCO (Società Italiana per lo Studio della Storia Contemporanea), del CESH (European Committee for Sports History) e della SISS (Società Italiana di Storia dello Sport). Fra le sue recenti pubblicazioni: Salvatore Contarini e la politica estera italiana (1891-1926), Roma, Aracne, 2017.

URL: < http://www.studistorici.com/progett/autori/\#Anastasi > 